\title{
ISOLATED TORSION OF HYDROSALPINX-A RARE PRESENTATION
}

Bharti Choudhary (Parihar), Aruna Kumar, Shabana Sultan

1. Assistant Professor. Department of Obstetrics \& Gynaecology, Sultania Zanana Hospital, Gandhi Medical College, Bhopal Madhya Pradesh.

2. Professor. Department of Obstetrics \& Gynaecology, Sultania Zanana Hospital, Gandhi Medical College, Bhopal Madhya Pradesh.

3. Assistant Professor. Department of Obstetrics \& Gynaecology, Sultania Zanana Hospital, Gandhi Medical College, Bhopal Madhya Pradesh.

\section{CORRESPONDING AUTHOR:}

Dr. Bharti Choudhary (Parihar),

115/5,OPD Block,

Sultania Zanana Hospital Campus,

Bhopal, Madhya Pradesh- 462001.

E-mail: drbhartiparihar@gmail.com

ABSTRACT: Isolated unilateral torsion of Hydrosalpinx is an infrequent but significant cause of acute lower abdomen pain in a female of reproductive age. The diagnosis is often difficult $\&$ is established during laparotomy or laparoscopy. A 38 year old Para 2 presented with lower abdominal pain \& acute retention of urine. Clinical \& Ultrasonographic findings suggested the diagnosis of torted left sided ovarian cyst. Laparotomy revealed a left sided torted Hydrosalpinx. Torsion of Hydrosalpinx is a rare event but should be included in differential diagnosis of lower abdominal pain in any female of reproductive age, particularly when they have a history of tubal sterilization and or PID.

KEY WORDS: Hydrosalpinx, torsion, Acute Abdomen

INTRODUCTION: Isolated torsion of the fallopian tube is a rare event. Isolated torted hydrosalpinx was first reported in 1890 by Bland-Sutton. The overall incidence has been reported as 1 in 1.5 million women. ${ }^{1}$. The condition more commonly involves the right side. The clinical and imagine findings are often non specific and this entity is difficult to diagnose preoperatively and this can lead to delay in instituting the correct management. Preoperative diagnosis was made in less than $20 \%$ of reported cases ${ }^{2}$.

CASE REPORT: A 38 yrs old para 2 was admitted on 09/11/2009 at 12:30 PM with history of pain and lump in abdomen since two month. There was history of acute retention of urine seven days prior to admission to our hospital for which she went to urologist who refer the patient to us with diagnosis of ovarian cystadenoma on ultrasonography.

On examination she was afebrile and normotensive. Palpation revealed lump in suprapubic region extending towards right iliac fossa.

Per vaginal examination revealed an anteverted normal sized uterus, deviated to left and there was a tender cystic mass of $8 \times 8 \mathrm{~cm}$ size on right side.

Her routine investigations were normal. Ultrasound revealed a right sided cystic mass with septation of $10 \times 8 \times 6 \mathrm{~cm}$. with small subserous fibriod of $2.4 \times 3.5 \mathrm{~cm}$. on anterior wall. Her serum CA-125 level was 5.4 unit/ml (within normal range).

Patient was taken for laparotomy on 20/11/09 at 11:45 AM which revealed right sided large hydrosalpinx $10 \mathrm{x} 8 \mathrm{~cm}$, torted with two twists, purple pink in colour with grossly normal 
ovary. Left tube, ovary and uterus were normal. Total abdominal hysterectomy and bilateral salpingo oophorectomy was performed. Post operative course was uneventful.

DISCUSSION: Isolated tubal torsion is a rare but a significant cause of lower abdominal pain. Although the exact cause for tubal torsion is not known, many etiologies have been proposed, these includes anatomical abnormalities (long mesosalpinx, tubal abnormalities, haematosalpinx, hydrosalpinx, hydatids of Morgagni), Physiological abnormalities (abnormal peristalsis, hypermotility of tube, tubal spasm and intestinal peristalsis), Haemodynamic abnormalities (venous congestion in the mesosalpinx), Sellheim theory (sudden body position change.), Trauma, previous surgery or disease (tubal ligation or pelvic inflammatory disease.), Gravid uterus. $1,2,3$

All these factors contribute to the development of tubal torsion by providing a point of reference (fulcrum) around which the tube can twist. Hydrosalpinx is a predisposing factor in tubal torsion. Surgical division of the tube may weaken the structural support provided by the mesosalpinx resulting in greater motility and greater risk of torsion ${ }^{4}$

Many reports indicate that torsion of the hydrosalpinx is more common on the right side than the left $1,2,3$. This may be due to the presence of sigmoid colon on the left side causing limitation of tubal motility and also due to the slow venous flow on the right side, which may results in congestion hence torsion on right side.

Fallopian tube torsion is more common during reproductive age group. It rarely occurs

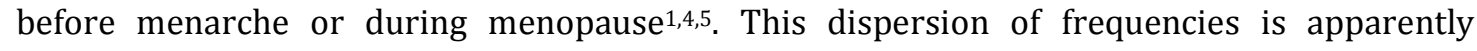
because most risk factors for tubal torsion occur mainly in the reproductive age group.

The most common presenting symptom is pain, which begins in the lower abdomen or pelvic on the affected side but may radiate to the flank or thigh ${ }^{1,2}$. The onset of pain is sudden and cramp like and may be intermittent ${ }^{3}$ It is frequently associated with nausea, vomiting, scanty uterine bleeding and occasionally urinary symptoms like frequency, urgency with voiding difficulties and rarely with retention of urine as in our case.

Preoperative diagnosis of twisted fallopian tube has not been possible due to physical findings associated with the other common diseases and non specificity of the imaging findings-A tenet that held true in this case as well. The ovarian cyst adenoma closely abutting the oedematous, twisted and dilated fallopian tube mimicked an ovarian lesion clinically and sonographically².

The differential diagnosis of fallopian tube torsion includes acute appendicitis, ectopic pregnancy, PID, twisted ovarian cyst or degenerative leiomyoma ${ }^{1,2,3}$.

The management is laparoscopic adenexal detorsion in the reproductive age group and complete resection with or without hysterectomy when the tissue is gangrenous, there is tubal or ovarian neoplasm or the women has completed her family3.

Torsion of hydrosalpinx is a rare but significant cause of acute lower abdominal pain in women of reproductive age. Clinician should consider tubal torsion in the differential diagnosis in women who exhibit pre disposing factors. Early surgical intervention is the treatment of choice.

\section{REFERENCE}

1. Bakshi R, Mehta A. Isolated torsion of fallopian tube with hydrosalpinx: A Rare presentation. The internet journal of gynecology and obstetrics. 2009 vol. 10-no 2 
2. Shukla R. Isolated torsion of the hydrosalpinx: a rare presentation, British Journal of Radiology 2004, 77,784-786

3. Krissi H, Shalev J et al. Fallopian tube torsion: Laparascopic evaluation and treatment of a rare gynaecological entity. J Am Board fam pract 2001: 14: 274-7 (S_).

4. Krissi H, Orivieto $\mathrm{R}$ et al. Torsion of fallopian tube following pomeroy tubal ligation: A rare case report and review of the literature. Euro. J. Obstet. Gynaecol. Reprod.biol.1997:17:107-9

5. Diaa E. E Rizk, Brinda Lakshminarasimha. Torsion of fallopian tube in an adolescent, Journal of pediatric adolescent gynecology 2000,15:159-161

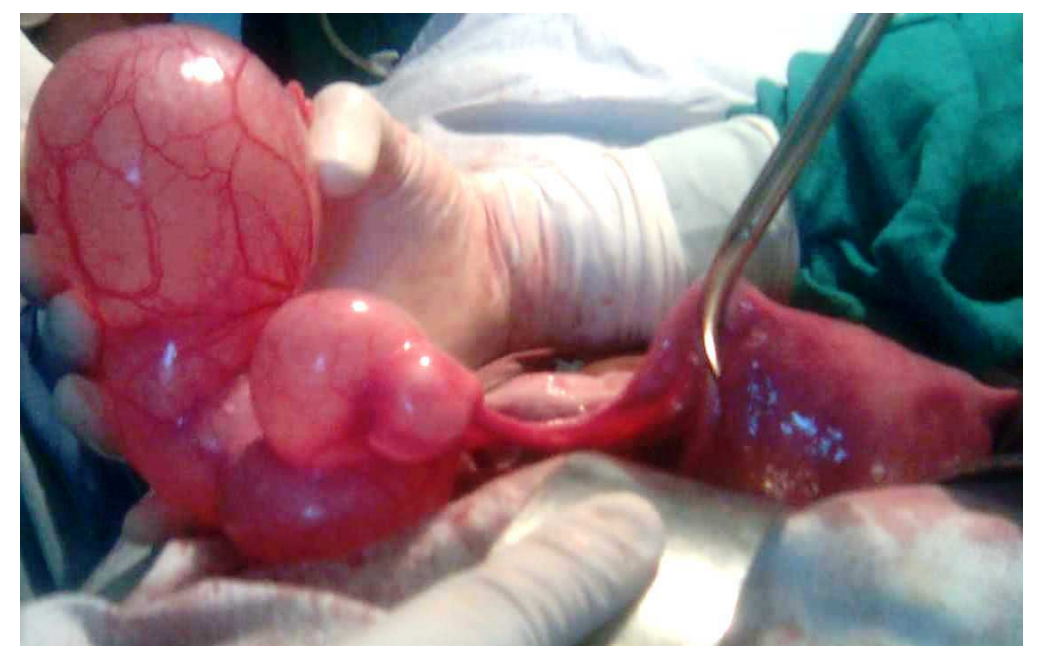

Figure1. Right sided hydrosalpinx measuring $10 x 8 \mathrm{~cm}$ with two twists, has been delivered out. The right ovary is seen separately with no twist of ovarian pedicle.

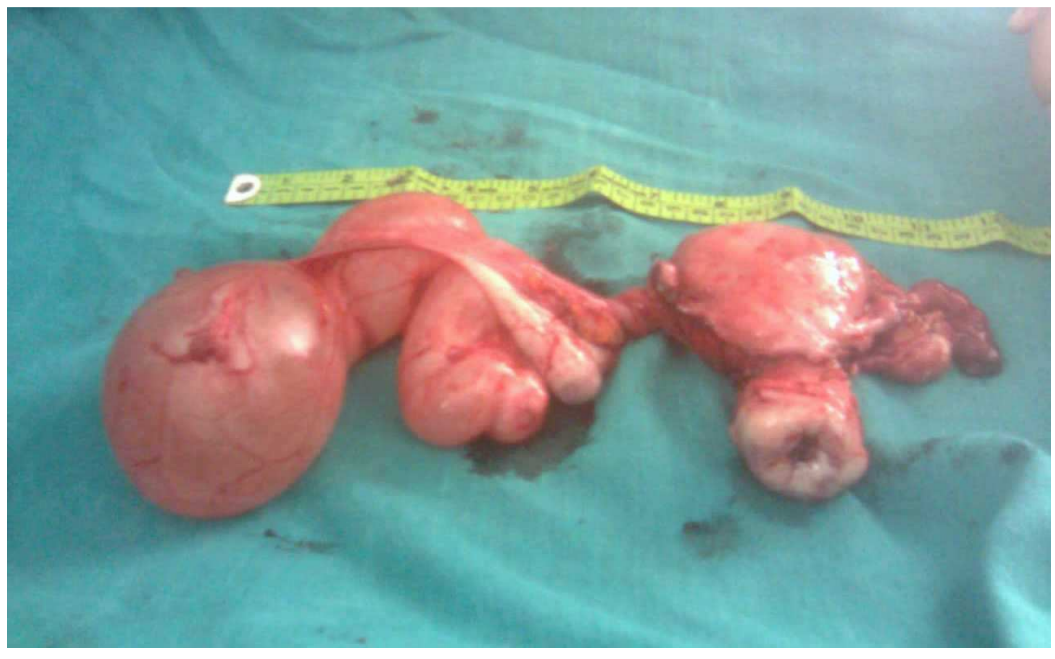

Figure2. Specimen of uterus with cervix with bilateral fallopian tubes and ovaries showing right sided twisted hydrosalpinx with two twists with grossly normal right sided ovary and left sided adnexa. 